\title{
Revealing electromigration on dielectrics and metals through the step-bunching instability
}

\author{
Victor Usov $\odot, 1,2,{ }^{*}$ Cormac Ó Coileáin, ${ }^{1,2,3}$ Alexander N. Chaika, ${ }^{4}$ Sergey I. Bozhko, ${ }^{4}$ Valery N. Semenov $\odot,{ }^{4}$ \\ Sergey Krasnikov $\odot,{ }^{1,2}$ Olzat Toktarbaiuly, ${ }^{1,2,5}$ Stoyan Stoyanov, ${ }^{6}$ and Igor V. Shvets ${ }^{1,2}$ \\ ${ }^{1}$ School of Physics, Trinity College Dublin, Dublin 2, Ireland \\ ${ }^{2}$ Centre for Research on Adaptive Nanostructures and Nanodevices (CRANN), Trinity College Dublin, Dublin 2, Ireland \\ ${ }^{3}$ School of Chemistry, Trinity College Dublin, Dublin 2, Ireland \\ ${ }^{4}$ Institute of Solid State Physics RAS, Chernogolovka, Moscow district 142432, Russian Federation \\ ${ }^{5}$ Nazarbayev University, 53 Kabanbay Batyr Avenue, Astana 010000, Kazakhstan \\ ${ }^{6}$ Institute of Physical Chemistry, Bulgarian Academy of Sciences, 1113 Sofia, Bulgaria
}

(Received 3 July 2019; revised 5 June 2020; accepted 12 June 2020; published 6 July 2020)

\begin{abstract}
Electromigration, due to its technological and scientific significance, has been a subject of extensive studies for many years. We present evidence of electromigration in dielectric materials, namely $C$-plane sapphire, obtained from direct experimental observation of an atomic step-bunching instability driven by electromigration. We further expand upon our previously reported findings of electromigration induced step-bunching transformation of a metal surface. The only system where electromigration driven step bunching has been observed and comprehensively investigated is the low index surfaces of silicon. In this study we show that electromigration driven SB can be induced on a variety of crystallographic surfaces, including metals and insulating oxides, and may be more prevalent than previously thought. Electric fields were applied at high temperature to W(110) and $\mathrm{Al}_{2} \mathrm{O}_{3}(0001)$ crystals whereupon their surface reordered to a morphology closely resembling that of $\mathrm{Si}(111)$ with atomic steps bunched by electromigration. This suggests that the mechanism of step bunching on the W(110), $\mathrm{Al}_{2} \mathrm{O}_{3}(0001)$, and $\mathrm{Si}(111)$ can be fundamentally the same. Annealing W(110) offcut in the [001] direction with an up-step current produced a morphology with the bunch edges composed of zigzag segments meeting at a right angle.
\end{abstract}

DOI: 10.1103/PhysRevB.102.035407

\section{INTRODUCTION}

Mass transport under the influence of an applied electric field has been a subject of extensive studies for decades due to its technological and scientific significance. It has an impact in many fields including electrophoresis, resistive switching, electrochemistry, and electromigration [1-9]. Electromigration is driven by continuous momentum transfer from electrons to the lattice ions, known as the electron wind $[8,9]$, or by direct electrostatic force on surface atoms with an uncompensated positive effective charge [10]. The former dominates in metals and results in formation of voids and hillocks which are mainly responsible for interconnect failures in integrated circuits [9]. The latter is known to produce significant transformations of surface morphology by means of a coarsening step-bunching instability. The step-bunching instability transforms vicinal surfaces of single crystals by aggregating monoatomic steps into micron-scale step bunches comprised of tens or hundreds of densely packed atomic steps separated by wide flat terraces. Step bunching (SB) driven by electromigration has been extensively studied since its discovery although the only system where it was has been conclusively observed was the vicinal low index surfaces of silicon [11], especially $\mathrm{Si}(111)$, which became the prime sys-

*Corresponding author: usovv@tcd.ie tem for studying SB instabilities. In this paper we demonstrate that under the appropriate conditions electromigration can manifest itself in terms of coarsening SB on vicinal surfaces of metals and dielectrics.

Vicinal crystal surfaces play an important role in technological applications and in condensed-matter studies because atomic steps on these surfaces affect dynamics of multiple processes such as atom or molecule adsorption, thin-film or crystal nucleation and growth, and surface chemical and catalytic reactions [12-14]. Vicinal surfaces of high uniformity and regularity can be created simply by offsetting the surface of a single crystal by a small angle from a low index crystallographic plane. This has led to the widespread use of vicinal surfaces in solid-state studies as they provide atomically uniform nanostaircase structures that can be created at length scales which are not feasible with conventional lithography techniques [15].

Vicinal surfaces can also be used to control surface reactivity, as atoms at the step edge and kink positions have lower bond saturation than atoms on terraces and can potentially demonstrate increased reactivity [16]. Also, substrate atomic steps provide favorable positions for adatoms during epitaxial film growth, which makes vicinal surfaces ideal as templates for directing ordered growth of thin films and nanowire arrays [17-20]. In addition, the stepped morphology of surfaces creates active sites for catalytic reactions to occur at the step edges. For example the $\mathrm{N}_{2}$ dissociation on $\mathrm{Ru}(0001)$ is wholly 
dominated by atomic steps with the adsorption rate at the steps being at least nine orders of magnitude higher than on terraces at $500 \mathrm{~K}$ and the corresponding difference in activation energy is $1.5 \mathrm{eV}$ [21]. Furthermore, catalytic reductions or molecule dissociation predominantly occur at particular sites and stepped surfaces of metal oxides can expose chemically diverse surface terminations [22,23]. Aside from catalytic reactions, vicinal surfaces allow a degree of anisotropy to be introduced into magnetic properties of heteroepitaxial ultrathin films. Atomic steps locally break films' rotational symmetry forcing additional in-plane step-induced uniaxial anisotropy between magnetically equivalent crystallographic directions or can rotate the original magnetic easy axes towards other directions [24-28].

Confinement of atomic steps to smaller areas can be potentially advantageous for many applications that rely on the size, shape, height, and interstep distance [29-37]. In this regard, SB driven by surface electromigration is of particular interest as it provides an additional degree of dynamic control to modify the surface structure through the strength and orientation of the applied electric field in addition to various process parameters such as annealing temperature and time, the heating and cooling rates, and slope and crystallographic direction of the miscut [37-41]. However, the exclusive focus of studies on the SB phenomenon to silicon has limited its research use and potential for applications.

In this study we show that electromigration driven SB can be induced on a variety of crystallographic surfaces, including metals and insulating oxides. This is demonstrated on $\mathrm{W}(110)$ and $\mathrm{Al}_{2} \mathrm{O}_{3}(0001)$ where surface morphologies created by electric field at high temperature closely resemble $\mathrm{Si}(111)$ with atomic steps bunched by electromigration, suggesting that the mechanism of SB on Si(111), W(110), and $\mathrm{Al}_{2} \mathrm{O}_{3}(0001)$ can be fundamentally the same. The similarities include, in particular, the scaling relationships between the step bunch height and its slope, atomic steps crossing wide terraces gradually changing shape towards formation of antibands, the existence of a critical field $\left(E_{\mathrm{cr}}\right)$ below which the step-bunching process ceases, and dependence of the SB morphology on the miscut direction [40-42].

We discuss a unique feature of SB behavior on W(110). It is well known that $\mathrm{SB}$ on $\mathrm{Si}$ has quite complex temperature and electric current orientation dependencies [11,38-44]. In particular, SB can be realized on $\mathrm{Si}(111)$ by electric current driven along the miscut in only one particular direction, while the surface remains stable against step bunching when the current is reversed. Moreover, annealing a bunched surface with current of the opposite direction results in de-bunching, i.e., dissociation of step bunches towards a single step configuration. We observed this on $\mathrm{Al}_{2} \mathrm{O}_{3}(0001)$ but discovered that on W(110) SB takes place for both up- and down-step directions of direct current.

\section{EXPERIMENTAL PROCEDURE}

SB on sapphire and tungsten became possible due to a specially designed experimental vacuum setup (base pressure of $2 \times 10^{-10}$ Torr) combining independent thermal annealing and voltage control across the sample [44]. The heat is supplied by an empty effusion cell employed as a heating shroud, surrounding a customized sample holder connected to a dc power supply. The annealing temperature was measured and controlled by a thermocouple placed next to the crucible.

Sapphire $10 \times 1.5 \times 0.5 \mathrm{~mm}$ rectangular strips were cut from single-crystal $C$-plane $\mathrm{Al}_{2} \mathrm{O}_{3}$ substrates (MTI Corp.) with a $2^{\circ}$ miscut off the (0001) plane in the [1-210] direction, with the long side aligned to the miscut direction. Samples were sonicated in acetone, isopropanol, and ethanol before they were mounted on a sample holder and transferred into the annealing chamber. The sample temperature was gradually raised to $1500{ }^{\circ} \mathrm{C}$ and maintained for several hours. Voltages to create electric fields $E$ from 0 to $140 \mathrm{~V} / \mathrm{cm}$ were applied along the miscut in either the up- or down-step direction.

Preparation of tungsten samples was much more complex and involved a multistep fabrication process. First, a singlecrystal W ingot with dislocation density of $2 \times 10^{4} \mathrm{~cm}^{-2}$ was grown by the floating zone technique. Small-angle boundaries and dislocations were removed from the ingot by subjecting it to an overcritical plastic deformation, followed by recrystallization at high temperature. Lattice parameters were monitored by x-ray diffraction and rocking curves with 50 angular seconds at full width at half maximum were recorded for the (110) reflection $\left(\mathrm{Cu} K_{\alpha}\right) .10 \times 1.5 \times 0.5 \mathrm{~mm}$ rectangular strips were cut by spark cutting at different miscut angles $\beta$ off the $\mathrm{W}(110)$ surface with the long side along the miscut direction $\left(\beta=0.8^{\circ}, 1.1^{\circ}\right.$, and $2.6^{\circ}$ in the [1-1-2] and $\beta=$ $0.8^{\circ}$ in the [001] direction). The strips were polished with SiC 240-2000-grit sandpapers on the top side and 240-600 grit on the flip side, and finished by electropolishing cycles of $30 \mathrm{~s}$ at $15 \mathrm{~V}$ in $2 \% \mathrm{NaOH}$ solution. Cleaning was performed in a designated ultrahigh vacuum (UHV) system with a base pressure $2 \times 10^{-10}$ Torr to remove carbon impurities prior to sample transfer to the annealing setup. The cleaning process consisted of alternate cycles of oxidation and flash annealing in an electron bombardment heater. During each cycle, the surface was first annealed at $1300{ }^{\circ} \mathrm{C}$ (measured with an infrared pyrometer Ircon UX20P, emissivity 0.35) in a $1 \times 10^{-6}$ Torr oxygen atmosphere for $60 \mathrm{~min}$. The resulting oxide layer was then removed from the surface by repeated flash annealing to $2100{ }^{\circ} \mathrm{C}$ for 15 -s intervals. The pressure rose to $1 \times 10^{-8}$ Torr during the flashes but rapidly recovered to the base pressure. This procedure was repeated until carbon and oxygen impurity levels were below the auger electron spectroscopy detection limit $(<1$ at. \%) and the surface produced a sharp $(1 \times 1)$ low-energy electrondiffraction (LEED) pattern, consistent with the bulk termination of vicinal W(110). Surface morphology was analyzed in situ by scanning tunneling microscopy (STM). Finally, a protective $\mathrm{WO}_{2}$ layer was grown by annealing samples at $1200^{\circ} \mathrm{C}$ in oxygen atmosphere at $P=5 \times 10^{-7}$ Torr for $60 \mathrm{~min}$ before removing them from UHV.

These samples were mounted on a holder between two electrical contacts and placed into the annealing chamber where they were first outgassed at $700{ }^{\circ} \mathrm{C}$ and then the protective oxide was removed by annealing at $1300^{\circ} \mathrm{C}$ for $1 \mathrm{~h}$. Finally, the crucible temperature was gradually raised to $1500^{\circ} \mathrm{C}$ and the SB morphology was produced by passing 6- or 12-A direct current through the samples for several hours along their long side perpendicular to the orientation of the atomic steps. A high annealing temperature was chosen 
in order to ensure high mobility of the surface adatoms but still considerably lower than the melting point of tungsten at $3422^{\circ} \mathrm{C}$. After cooling to room temperature, samples were removed from UHV for ex situ atomic force microscopy (AFM) characterization.

\section{RESULTS AND DISCUSSION}

\section{A. Step bunching on $\mathrm{Al}_{2} \mathrm{O}_{3}(0001)$}

The nature of dielectric materials is to prohibit direct currents and it is not intuitive that any effect of electromigration should be observed in such systems, as opposed to the integrated circuits where electromigration results in the clear formation of voids and hillocks. However, with the assumption that atoms on a crystalline insulating surface can develop an effective charge at high temperatures, an applied electric field may be sufficient to bias surface atom diffusion and electromigration may be manifested through SB transformation of the surface morphology. In this regard, highly ionic solids, such as alphasapphire $\left(\alpha-\mathrm{Al}_{2} \mathrm{O}_{3}\right)$, are particularly attractive as most of them are electrically insulating and their surface atoms are likely to have an uncompensated effective charge at high temperatures. The basal plane of $\alpha-\mathrm{Al}_{2} \mathrm{O}_{3}$ appeared to be an excellent candidate for studying effects of electromigration on dielectrics due to its high melting point $\left(2030^{\circ} \mathrm{C}\right)$, low volatility, and high electrical resistivity of $10^{16} \Omega \mathrm{cm}$.

In $\alpha-\mathrm{Al}_{2} \mathrm{O}_{3} \mathrm{O}^{2-}$ anions are organized in a hexagonal-closepacked lattice while $\mathrm{Al}^{3+}$ cations occupy two of the three available octahedrally coordinated interstitial sites between oxygen planes [45-47]. Oxygen and aluminum planes form an $R$-Al-Al-O-Al-Al-O- $R$ stacking sequence in the [0001] direction with relatively well separated adjacent $\mathrm{Al}$ layers $(R$ denotes structure in perfect registry with the bulk). The (0001) surface can be created by breaking the sequence at one of three possible terminations of which the $\mathrm{Al}$ termination, $\mathrm{Al}-\mathrm{O}-\mathrm{R}$, with $\mathrm{Al}$ in one of three octahedral sites, is energetically the most stable [48-56]. The high surface free energy of the bulklike $\mathrm{Al}$ termination is lowered by the inward relaxation of aluminum ions towards the underlying oxygen layer where the $\mathrm{Al}^{3+}$ positive charge is screened more effectively [45,46,5761].

Heating to $1500{ }^{\circ} \mathrm{C}$ in UHV $\mathrm{Al}_{2} \mathrm{O}_{3}(0001)$ undergoes several intermediate reconstructions before it achieves a $(\sqrt{ } 31 \times$ $\sqrt{ } 31) R \pm 9^{\circ}$ reconstruction [62-66], which is stable under further annealing and resistant to reoxidation under vacuum conditions [64,67-69]. $R \pm 9^{\circ}$ denotes a $9^{\circ}$ tilt with respect to the $C$ axis of sapphire. The onset temperature of the $(\sqrt{ } 31 \times$ $\sqrt{ } 31) R \pm 9^{\circ}$ reconstruction varies in studies between 1000 and $1400{ }^{\circ} \mathrm{C}[63,64,70,71]$ supposedly due to differences in surface preparation prior to UHV annealing [72]. The $(\sqrt{ } 31 \times$ $\sqrt{ } 31) R \pm 9^{\circ}$ reconstruction evolves via sublimation of the two topmost $\mathrm{O}$ planes and reorganization of three unconstrained topmost Al layers into a well-ordered hexagonal-close-packed surface structure with an interatomic distance of $0.30 \mathrm{~nm}$. Atoms in the two lower $\mathrm{Al}$ layers remain at their octahedral sites and complete the Al-rich stoichiometric termination (Al-Al-O-R). The Al-rich termination contains three hexagonal sublattices of highly symmetric bonding sites that define the hexagonal symmetry of the $\mathrm{Al}$ surface, being reflective of the symmetry of the $\mathrm{Si}(111)$. At the same time, periodic
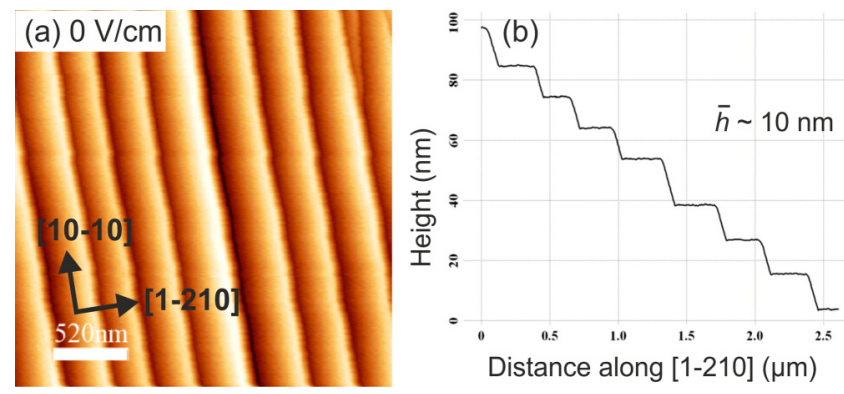

FIG. 1. (a) AFM image of the $\mathrm{Al}_{2} \mathrm{O}_{3}(0001)$ surface annealed at $1500{ }^{\circ} \mathrm{C}$ at $P=5 \times 10^{-9}$ Torr with zero electric field $(E=0 \mathrm{~V} / \mathrm{cm})$. Upon annealing the surface transforms into (0001) and (1-216) facets with the edges running along [10-10]. Scale bar is $520 \mathrm{~nm}$. (b) Cross sectional profile along the offcut [1-210] direction of the same surface locally plane-fitted to one of the (0001) facets. Average "step" height is $\sim 10 \mathrm{~nm}$.

switching of $\mathrm{Al}$ bonding positions between the three sublattices results in the formation of a hexagonal superstructure characterized by a $2.65 \mathrm{~nm}$ rhombic unit cell $[64,69,73-75]$, composed of three different types of Al stacking domains. Each domain type is composed of $\mathrm{Al}$ cations ionically bonded to oxygen at a particular type of bonding site: either at triangular voids above $\mathrm{O}$, voids above $\mathrm{Al}$, or directly near the top of $\mathrm{O}$ atoms [74].

The $(\sqrt{ } 31 \times \sqrt{ } 31) R \pm 9^{\circ} \mathrm{Al}_{2} \mathrm{O}_{3}(0001)$ reconstructed surface is broadly accepted as being insulating and benchmarking experimental studies of this system have been conducted using techniques unaffected by the insulating character of the surface $[69,73,74]$. Although some computational studies predict possible metallic states in the $(\sqrt{ } 31 \times \sqrt{ } 31) R \pm 9^{\circ}$ surface layer [60] there is no conclusive experimental evidence to support this claim $[76,77]$ and a conduction current in the system is virtually impossible. At the same time, an electric field can influence the $\mathrm{Al}$ atoms due to their positive charge developed by redistribution of the electronic density between $\mathrm{Al}$ and underlying $\mathrm{O}$ plane. Thus, an electric field above a certain critical strength can be sufficient to cause surface electromigration of $\mathrm{Al}$ cations in the direction of the applied field and in doing so destabilize the surface against step bunching.

Figure 1 shows an AFM image of a control sapphire sample thermally annealed at $1500{ }^{\circ} \mathrm{C}$ in vacuum in the absence of an electric field where the surface transformed into periodic $\sim 350$-nm-wide (0001) terraces and $\sim 10$-nmwide (1-216) facets with the edges running along the [10-10] direction. Such morphology is consistent with previous studies of $\mathrm{Al}_{2} \mathrm{O}_{3}(0001)$ surface faceting, and once established is known to be thermodynamically stable and not evolve with annealing time [78,79]. However, annealing with an $E$ field of $140 \mathrm{~V} / \mathrm{cm}$ applied along the miscut in the up-step direction creates a radically different morphology characterized by $1.5-2.0-\mu \mathrm{m}$-wide flat terraced regions separated by step bunches with a high density of atomic steps, $0.15-0.2 \mu \mathrm{m}$ wide and up to $100 \mathrm{~nm}$ high (Fig. 2). Notably wider, up to 4 $\mu \mathrm{m}$, terraces were also observed. The authors are not aware of any existing studies that report a change in the surface evolution process from faceting to step bunching induced by 


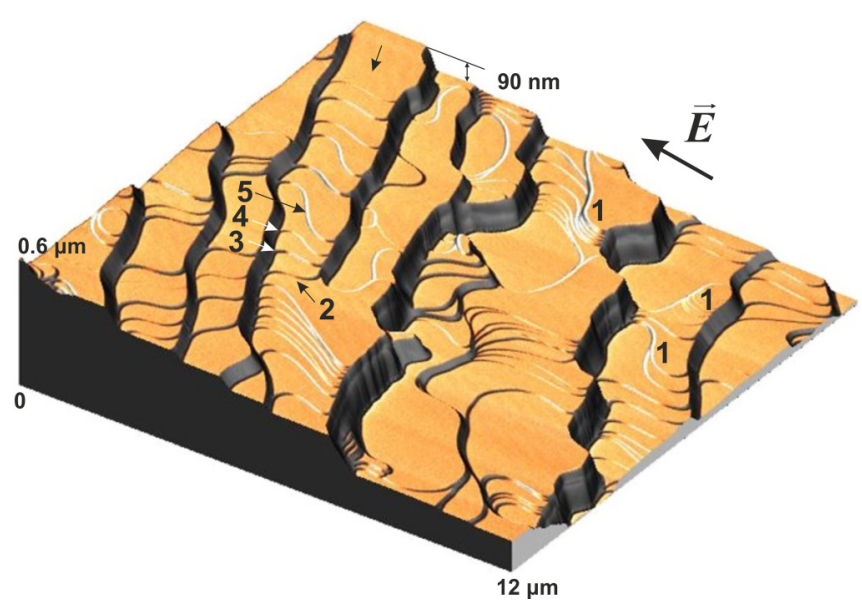

FIG. 2. $11 \times 11 \times 0.6 \mu \mathrm{m}$ AFM image of $\mathrm{Al}_{2} \mathrm{O}_{3}(0001)$ annealed at $1500{ }^{\circ} \mathrm{C}$ with $E=140 \mathrm{~V} / \mathrm{cm}$ applied in the up-step direction $\left(P=5 \times 10^{-9}\right.$ Torr $)$. Surface morphology comprises alternating 1.5-2- $\mu \mathrm{m}$-wide terraces and 150-200-nm-wide step bunches with a height up to $100 \mathrm{~nm}$. A wider 4- $\mu \mathrm{m}$ terrace is also captured in the image. 1 marks antibands, i.e., secondary step bunches with slopes opposite to the slopes of primary bunches. An arrow marks a terrace that captures evolution of individual crossing atomic steps towards an antiband formation as the terrace widens: 2 is an atomic step curved in a long-S shape, 3 is a step in a steady state $S$ shape, 4 is a step that developed a segment in the middle parallel to the step-bunch edges indicating the onset of an antiband, 5 marks progression towards formation of an antiband.

electric field. The transformed $\mathrm{Al}_{2} \mathrm{O}_{3}(0001)$ surface closely resembles the step-bunching morphology previously observed on $\mathrm{Si}(111)$ which is produced by a fine balance between biased atomic drift, atomic adsorption and desorption, step-step repulsive interaction, and attachment-detachment kinetics at the steps. Quantitatively this morphology is described by the scaling relationship

$$
y_{m} \sim h^{\alpha} E^{q}
$$

between the maximum slope of a bunch $y_{m}$, the step bunch height $h$, and the electric field $E$ ( $\alpha$ and $q$ are positive scaling exponents) [38-42,80]. For silicon SB morphology can be produced, depending on the temperatures interval, by either up- or down-step electromigration with $E$ fields of several $\mathrm{V} / \mathrm{cm}$, which is significantly lower than the $E$ fields used in this study [11,38-40,81]. For the reconstructed $C$-plane sapphire, the sign of the effective charge at $1500^{\circ} \mathrm{C}$ has not been conclusively established, yet it is reasonable to propose that the sign is positive and the step bunching is driven by the up-step electromigration of $\mathrm{Al}$ cations in the direction of the applied electric field. We propose that detachment of $\mathrm{Al}$ atoms from the steps, facilitating the step movement, is accompanied by sublimation of underlying $\mathrm{O}$ and development of the $(\sqrt{ } 31 \times \sqrt{ } 31) R \pm 9^{\circ}$ reconstruction on the vacated $\mathrm{Al}$ sites which become part of a widening terrace.

Equation (1) implies that the bunch slope varies along the miscut direction and reaches a certain maximum which increases as the height of the bunch increases. The same correlation between the height and the slope was found for the $\mathrm{Al}_{2} \mathrm{O}_{3}(0001)$ as demonstrated in Figs. 3(a) and 3(b) which
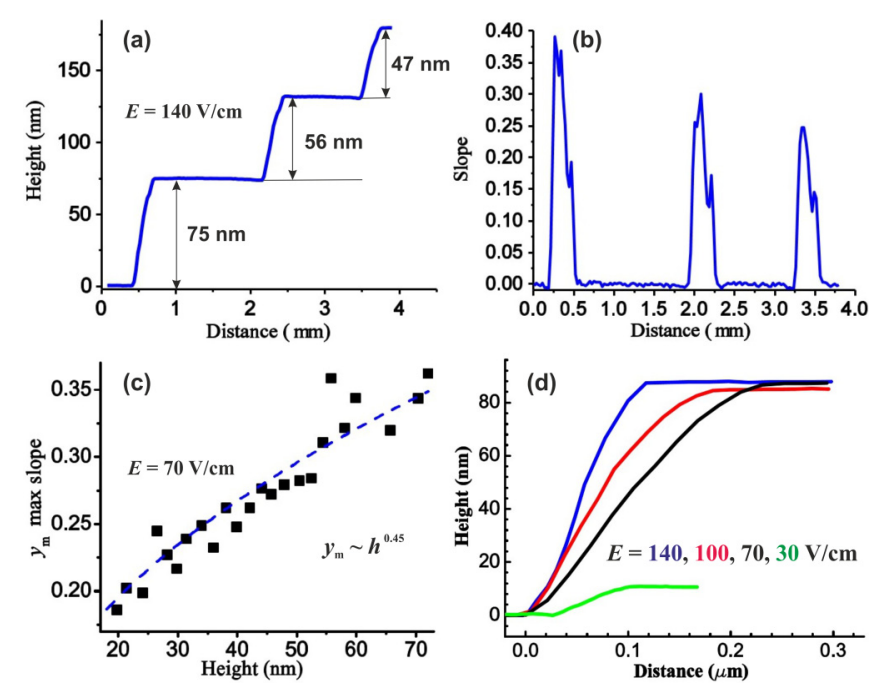

FIG. 3. (a) Profile and (b) the slope variation of the $\mathrm{Al}_{2} \mathrm{O}_{3}(0001)$ along the same line along the offcut. Annealing conditions: $T=$ $1500^{\circ} \mathrm{C}, E=140 \mathrm{~V} / \mathrm{cm}$ in the up-step direction, $P=5 \times 10^{-9}$ Torr. The higher slope maxima are located towards the middle of the higher bunches. (c) Maximum slope $y_{m}$ as a function of the bunch height $h . y_{m}$ increases with $h$. (d) Line profiles along the offcut direction for the 82-84-nm-high step bunches produced by annealing $\mathrm{Al}_{2} \mathrm{O}_{3}(0001)$ with the varying strengths of the $E$ field. Increasing the $E$ fields from 70 to $140 \mathrm{~V} / \mathrm{cm}$ results in the tighter compression of the monatomic steps and steeper bunch slopes. Annealing at $E=$ $30 \mathrm{~V} / \mathrm{cm}$ produced a faceted $\mathrm{Al}_{2} \mathrm{O}_{3}(0001)$ surface indistinguishable from morphology obtained by thermal annealing alone (Fig. 1). The cessation of SB indicates that the $E$ field has reached the critical value $E_{\text {cr }}$, which for $\mathrm{Al}_{2} \mathrm{O}_{3}(0001)$ lies between 30 and $70 \mathrm{~V} / \mathrm{cm}$ at $1500^{\circ} \mathrm{C}$.

shows that the slope varies along the offcut direction and the higher maxima are somewhere towards the middle of the higher bunches. For initial quantitative analysis the maximum slope $y_{m}$ was plotted against the bunch height $h$ [Fig. 3(c)] and a clear trend was observed, with $y_{m}$ increasing with $h$. The $y_{m}(h)$ data were fitted to the $y_{m} \sim h^{\alpha}$ function and although the observed trend follows the power law, the scaling exponent $\alpha=0.45 \pm 0.06$ is lower than the theoretically and experimentally determined $\alpha=0.6$ for up-step or $\alpha=0.66$ for down-step electromigration on $\mathrm{Si}(111)[39-42,80]$. Although the graph in Fig. 3(c) provides strong evidence for the ascending trend of the $y_{m}(h)$ dependence, more data and a broader range of the bunch heights is desirable for an accurate measurement of the height scaling exponent.

The other aspect of Eq. (1) concerns the relationship between the $y_{m}$ and the $E$ field, which is known for bunched $\mathrm{Si}(111)$. It asserts that higher slopes are created by applying stronger electric fields during annealing. Sapphire revealed this trend, demonstrated in Fig. 3(d), which shows line profiles along the miscut direction for 82-84-nm high step bunches produced by annealing the $\mathrm{Al}_{2} \mathrm{O}_{3}(0001)$ with a variety of $E$ field strengths. Clearly, increasing the applied $E$ fields from 70 to $140 \mathrm{~V} / \mathrm{cm}$ results in the formation of steeper bunches due to greater compression of atomic steps. However, annealing with an $E$ field of $30 \mathrm{~V} / \mathrm{cm}$ was not sufficient to initiate an appropriate directional drift of surface adatoms, and a faceted $\mathrm{Al}_{2} \mathrm{O}_{3}(0001)$ surface was produced, similar to that 


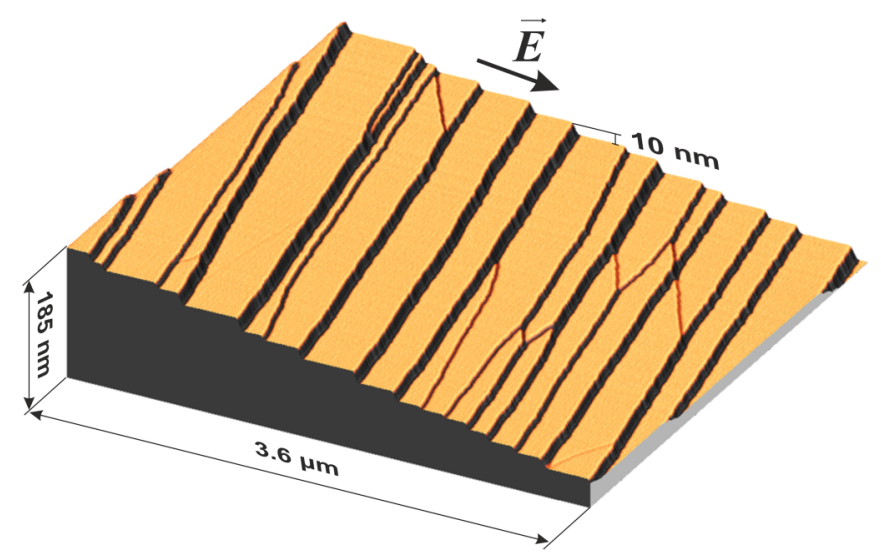

FIG. 4. $3.6 \times 3.6 \times 0.185-\mu \mathrm{m}$ AFM image of $\mathrm{Al}_{2} \mathrm{O}_{3}(0001)$ annealed at $1500{ }^{\circ} \mathrm{C}$ with $E=140 \mathrm{~V} / \mathrm{cm}$ applied in the down-step direction $\left(P=5 \times 10^{-9}\right.$ Torr $)$. Annealing creates faceted surface that cannot be distinguished from morphology created by thermal annealing alone (Fig. 1).

obtained for thermal annealing (Fig. 1). This cessation of bunching indicates the $E$ field has reached the critical value $E_{\text {cr }}$, which is an important characteristic of the SB instability [81]. From this experiment the critical field for $\mathrm{Al}_{2} \mathrm{O}_{3}(0001)$ lies between 30 and $70 \mathrm{~V} / \mathrm{cm}$ at $1500^{\circ} \mathrm{C}$, which is at least a factor of 60 greater than $E_{\text {cr }}$ for up-step and 20 for downstep electromigration on $\mathrm{Si}(111)$ with the same degree of surface vicinality $[44,81]$, indicating either lower mobility or smaller effective charge (or both) for $\mathrm{Al}$ surface adatoms on reconstructed $\mathrm{Al}_{2} \mathrm{O}_{3}(0001)$.

A distinctive feature of the $\mathrm{Si}(111)$ is its instability against SB for a specific direction of electromigration but stability for the opposing direction. Moreover, the application of an $E$ field in this opposing direction will result in debunching, i.e., widening of established step bunches and their gradual dissolution. To investigate whether this directional sensitivity of the SB instability exists on sapphire, vicinal $\mathrm{Al}_{2} \mathrm{O}_{3}(0001)$ was annealed under the same conditions but with the $E$ field applied in the opposite, down-step, direction. In this case, the sapphire exhibits faceted topography (Fig. 4) effectively indistinguishable from the morphology created by thermal annealing alone (Fig. 1). Furthermore, annealing a bunched surface with the opposing down-step $E$ field increases the interstep distance within the bunches resulting in their broadening (Fig. 5). This directional sensitivity to $E$ field matches the SB behavior on $\mathrm{Si}(111)$, indicating that the $\mathrm{SB}$ on $C$-plane sapphire is also driven by electromigration. Figure 5 shows AFM images of the same $\mathrm{Al}_{2} \mathrm{O}_{3}(0001)$ sample annealed in the presence of an $E$ field of $100 \mathrm{~V} / \mathrm{cm}$ first applied in the up-step [Fig. 5(a)] and then in the down-step direction [Fig. 5(b)]. After the second annealing, the step bunches dissociated and diminished the proportion of area occupied by terraces to such extent that the steps could be individually resolved [Fig. 5(b)]. Notably, steps isolated from the bunch are not monatomic but have heights between 1.1 and $10.8 \mathrm{~nm}$, i.e., include one or several $c$-lattice unit cells ( $1.29 \mathrm{~nm}$ high in the bulk).

Individual S-shaped steps, as shown in Fig. 2, crossing the wide terraces and not included in the step bunches, are also an important characteristic feature of surfaces step-bunched by

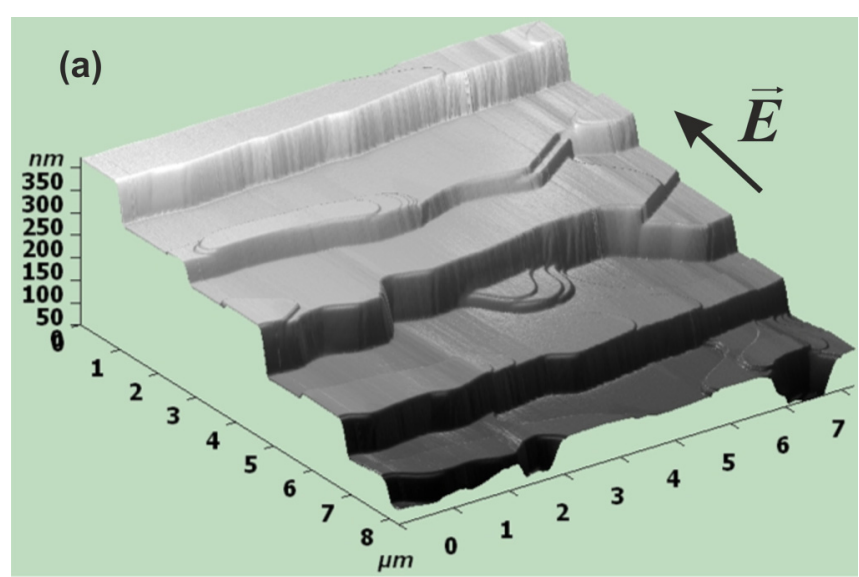

(b)

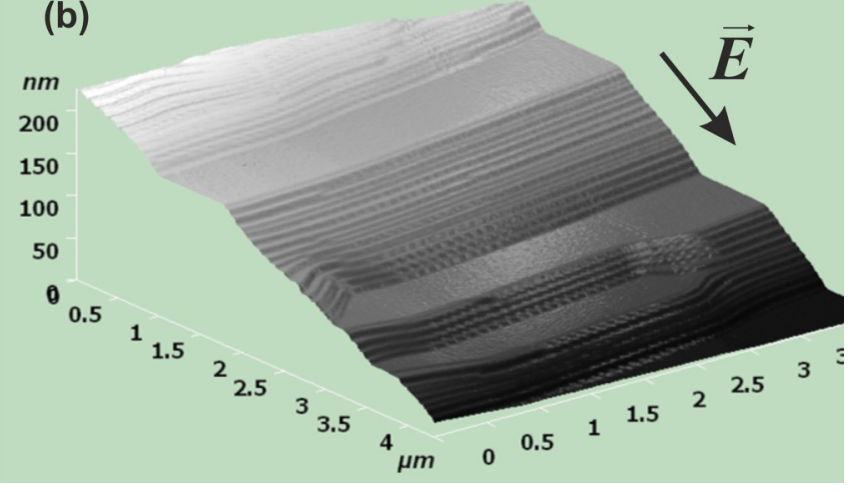

FIG. 5. AFM images of the same $\mathrm{Al}_{2} \mathrm{O}_{3}(0001)$ sample annealed at $T=1500{ }^{\circ} \mathrm{C}$ in the presence of the $E$ field of $100 \mathrm{~V} / \mathrm{cm}$ first applied in (a) the up-step and then (b) reversed in the down-step directions. Step bunches clearly occupy most surface after debunching and atomic steps can be clearly resolved. The width of an 85-nm-high bunch increased by nearly a factor of 4 from 220 to $980 \mathrm{~nm}$. This directional sensitivity to $E$ field matches the SB behavior on $\mathrm{Si}(111)$, indicating that the $\mathrm{SB}$ on sapphire $C$ plane is driven by electromigration.

electromigration. The shape evolution of these crossing steps gives rise to new morphologies such as antibands (marked 1 in Fig. 2), which can be described as secondary step bunches running along the terrace edges with slopes opposite to the surface global offcut direction [82-84]. As the terraces widen the shape of the crossing steps gradually develops in order to compensate for the gradient of the adatom concentration across terraces, via the appropriate variation of the step curvature [83-85]. Analysis of the shape can reveal the relative rates of adatom surface diffusion and adatom exchange with the crystal phase, and thus distinguish between the diffusion and the step-kinetic limited SB dynamics $[84,85]$. Notably, crossing steps on $\mathrm{Al}_{2} \mathrm{O}_{3}(0001)$ have heights predominantly from 2.4 to $6.5 \mathrm{~nm}$ corresponding to 2-5 $C$-lattice constants (within $0.2 \mathrm{~nm}$ error) which is much higher than the 0.2-0.3$\mathrm{nm}$ typical height of monatomic steps. Higher crossing steps, up to $\sim 10 \mathrm{~nm}$, were also detected closer to antibands and lower $0.4-0.5 \mathrm{~nm}$ further on flat terrace sections indicating the coalescence of individual crossing step upon their progression towards the antiband instability. In depth understanding of crossing steps dynamics upon their evolution towards 
formation of step bunches and antibands warrants future exploration with time resolved annealing experiments.

The terrace marked by an arrow in Fig. 2 captures all the essential stages of crossing steps evolution driven by electromigration as seen on $\mathrm{Si}(111)$ [84,85]. Initially, the crossing steps are curved in a long-S shape (marked 2), and in the terrace plane, the sign of their slope remains the same along the entire length. The adatom concentration gradient causes the crossing steps to recede along the terraces in an uneven manner towards a steady-state $\mathrm{S}$ shape (marked 3), which is characterized by alignment perpendicular to the step bunches and a change in sign of the slope in each half of the terrace. This symmetric $\mathrm{S}$ shape can only be maintained within a limited range of terrace widths, and as terrace widening progresses the step curvature cannot compensate for the adatom concentration gradient. From this point the steps lose their symmetry and develop a segment aligned parallel to the step bunches (marked 4) marking the onset of the antiband formation (marked 5). The onset and development of the antiband instability via evolution of crossing steps has been studied in detail on $\mathrm{Si}(111)$ exclusively for the down-step adatom drift [83-85] mainly due to a high SB rate which is faster by nearly two orders of magnitude as compared to the SB by the up-step electromigration $[38,40]$. In this case, terraces reach the width of $\sim 3.5-4.5 \mu \mathrm{m}$ in several minutes, sufficient for studies of the antiband instability. However, for up-step electromigration, a longer annealing time in the order of hours is required for terraces to reach about the same width and tens of hours to complete the antiband formation [81]. Antibands formed by the up-step electromigration were previously observed in the final stage of their development on $\mathrm{Si}(111)$ however, the resolution of individual crossing steps was beyond capabilities of the optical microscopy used in the study [86]. The criteria for the onset of the antiband instability has been theoretically established and experimentally examined for the down-step electromigration:

$$
L_{t}^{2} \frac{d_{s}\left|q_{\mathrm{eff}} E\right|}{\bar{\beta} l \Omega} \geqslant 8,
$$

where $d_{s}$ is the kinetic characteristic length, $\bar{\beta}$ is the step stiffness, $l$ is the initial interstep distance, $\Omega$ is the surface area of a single atomic site, $q_{\text {eff }}$ is the effective electric charge of surface adatoms, $L_{t}$ is the terrace width, and $E$ is the applied electric field [84]. Equation (2) suggests that the onset of the antiband instability can be potentially established on narrower terraces by higher applied $E$ field. However, this is impossible on silicon as the increased $E$ field results in the exponential growth of the heating current and fast transition to the next temperature regime where $\mathrm{Si}(111)$ is stable against $\mathrm{SB}$ or approaches the melting point of silicon $\left(1414^{\circ} \mathrm{C}\right)$. Sapphire, on the other hand, has very low conductivity and allows application of significant electric field without any risk of increasing the sample temperature and establish the earlier onset of the antiband instability such as in Fig. 2 where $L_{t} \approx$ $2 \mu \mathrm{m}$ is sufficient to initiate the antiband formation at $E=$ $140 \mathrm{~V} / \mathrm{cm}$. This is less than half that for $\mathrm{Si}(111)$ for down-step [83-85] and a factor of 5 for the up-step [86] electromigration. This opens fresh prospects for systematic studies of the electromigration induced surface instabilities and, possibly, an observation of new previously unknown surface morphologies at very high electric fields on narrow terraces at their limits of width $\left(L_{t}\right)$, as determined by the global surface offcut. It also remains a subject for future studies to determine the stability of vicinal $\mathrm{Al}_{2} \mathrm{O}_{3}(0001)$ against $\mathrm{SB}$ surface transformations for lower temperature reconstructions, and to establish whether change of temperature would require reversal of the direction of the destabilizing $E$ field, as is known for $\operatorname{Si}(111)$.

\section{B. Electromigration driven step bunching on W(110)}

Due to its technological implications, the most intensively studied effects of electromigration in metals to date is the growth of voids and hillocks caused by the electron wind, while electromigration driven SB effects have never been observed in such systems. It is widely accepted that for silicon, and now suggested for sapphire, that SB is a result of atomic migration caused by the direct electrostatic force acting on surface positive ions [10]. However, in the case of metals a significant direct force cannot be applied, as the samples act as effective shortcuts due to metal's low resistivity ( $\rho=48 \mu \Omega \mathrm{cm}$ at $1500{ }^{\circ} \mathrm{C}$ for tungsten) [87] resulting in only small applied voltages. Moreover, the effective direct force on atoms in metals is normally significantly reduced due to the field screening effect [88]. On these grounds, in contrast to silicon or sapphire, we expect the electron wind to be the driving force responsible for $\mathrm{SB}$ on tungsten, just as it is responsible for the known electromigration effects on metals.

In this study tungsten samples were annealed with direct currents of 6 or $12 \mathrm{~A}$ (current densities $j=0.8 \times 10^{7}$ or $1.6 \times 10^{7} \mathrm{~A} / \mathrm{m}^{2}$ respectively) and with applied electric fields of 0.04 or $0.08 \mathrm{~V} / \mathrm{cm}$ respectively, which is a factor of 15 and 7.5 less than the weakest field $E=0.6 \mathrm{~V} / \mathrm{cm}$ ever reported to induce a SB instability [44,81]. However, the magnitude of the effective charge in metals can be 10-30 times the elementary charge $(e)[89,90]$ and even several times larger again for step edge atoms [91,92] which is in contrast to $\mathrm{Si}(111)$ where the charge of surface atoms is only a fraction $[39,40,85]$ or at most double [84] of $e$. In this case, even for such low $E$ fields, an electromigration force of $1 \mathrm{eV} / \mathrm{cm}$ or higher can be easily achieved, which is known to be sufficient to induce and sustain the SB instability on $\mathrm{Si}(111)$ [81]. Additionally, a large localized force enhancement is known to be possible on metallic surfaces such as $\operatorname{Ag}(111)$ where the effective charge of atoms at the kink sites is nearly two orders of magnitude larger than of atoms on terraces $(1260 e$ vs $17 e$ respectively), which is mostly due to their reduced coordination and corresponding local redistribution of electronic density [93]. Such increased electromigration force can produce intensive atom detachment from the steps resulting in inevitable fluctuations of the interstep distances. Potentially, these fluctuations can grow in amplitude with the annealing time and lead to unknown surface transformations of metallic surfaces due to kinetic instabilities, which were previously thought to require onerously large current densities [94]. This was predicted in earlier studies [93] but it was only recently that the electromigration induced SB kinetic instability was demonstrated on W(110) [95], and now we develop on this finding in this section. 

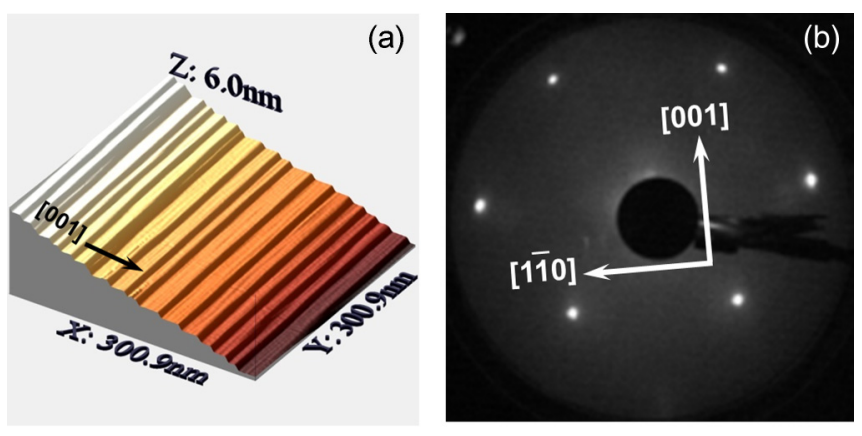

(c)
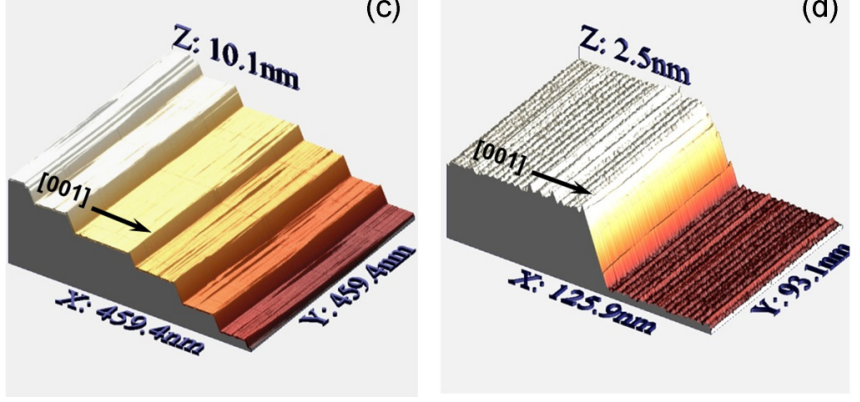

FIG. 6. (a) STM image and (b) LEED pattern (beam energy $62 \mathrm{eV}$ ) of a clean vicinal W(110), with a global off-cut of $0.8^{\circ}$ in the [001] direction, after cycled oxidation and flash-annealing in UHV. (c) STM image of an oxidized W(110) surface with the atomic steps agglomerated into $\sim 2$-nm-high bunches covered by a protective $\mathrm{WO}_{2}$ layer. (d) STM zoom-in image of an oxidized W(110). The oxide layer grows in the form of regular rows extending in the [001] direction, with a row periodicity of $3.0 \pm 0.1 \mathrm{~nm}$. Annealing in UHV at $1300^{\circ} \mathrm{C}$ removed the oxide layer and restored the monoatomic staircase surface topography as in [Fig. 6(a)].

We studied the manifestation of electromigration in metals via $\mathrm{SB}$ on the W(110) surface, which was chosen due to its quasihexagonal 2D lattice, similar to Si(111). Also, tungsten's high melting point allowed for annealing at $1500{ }^{\circ} \mathrm{C}$, which is the same temperature as was used for $\mathrm{SB}$ on $\mathrm{Al}_{2} \mathrm{O}_{3}(0001)$ and close to the temperature range $940-1390{ }^{\circ} \mathrm{C}$ required for $\mathrm{SB}$ on silicon. Figures 6(a) and 6(b) show the STM and LEED images of vicinal W(110), with a global off-cut of $0.8^{\circ}$ in the [001] direction, after cycled oxidation and flash annealing in UHV. The bright LEED pattern in Fig. 6(b) corresponds to a clean tungsten surface with a characteristic W(110) unit cell while the surface topography is characterized by monoatomic steps separated by W(110) terraces of unequal width varying between 20 and $50 \mathrm{~nm}$. Subsequent surface annealing in oxygen resulted in agglomeration of the atomic steps into $\sim 2-\mathrm{nm}$ high bunches covered by a protective $\mathrm{WO}_{2}$ layer [Fig. 6(c)] [96]. The STM [Fig. 6(d)] shows that the layer grows in the form of highly regular rows extending in the [1-10] direction parallel to the atomic steps, with an atomically precise row periodicity of $3.0 \pm 0.1 \mathrm{~nm}$. Annealing in UHV at $1300^{\circ} \mathrm{C}$ removed this oxide layer and restored the monoatomic staircase surface topography [Fig. 6(a)], which remained unchanged after a subsequent thermal annealing in UHV at $1500^{\circ} \mathrm{C}$. However, passing a $6 \mathrm{~A}\left(j=0.8 \times 10^{7} \mathrm{~A} / \mathrm{m}^{2}\right)$ current along the miscut in the up-step direction under the same conditions destabilized the surface and dramatically changed its morphology, which transformed into 1.0-3.0- $\mu \mathrm{m}$-wide flat W(110) terraces separated by 20-100-nm-high step bunches [Fig. 7(a)]. The same current passed in the opposite, downstep, direction transformed the monatomic steps into a stepdensity wave characterized by $2-2.5-\mathrm{nm}$-high step bands, comprised of only a few atomic steps and not increasing in height with annealing time [Fig. 7(b)]. Besides the bunchterrace morphology and sensitivity to current direction, the transformed W(110) also demonstrated other characteristic topological features associated with bunched Si(111), namely crossing steps, steeper slopes for higher bunches [Figs. 7(c) and 7(d)], and an associated power-law scaling relationship [95].

As would be expected from Eq. (1), a higher $I=12$ A annealing current driven in the up-step direction $(j=1.6 \times$ $10^{7} \mathrm{~A} / \mathrm{m}^{2}$ ) produced a bunched surface with steeper slopes [Fig. 7(e)] [95]. Notably however, the same current driven in the opposite, down-step, direction also destabilized the $\mathrm{W}(110)$ and produced a step-bunched surface morphology [Fig. 7(f)]. At present, the surface instability against SB for both up- and down-step currents at a single temperature is unique to $\mathrm{W}(110)$ and contrasts with $\mathrm{Si}(111), \mathrm{Al}_{2} \mathrm{O}_{3}(0001)$, and $\mathrm{W}(110)$ at lower current densities, where the SB has only been observed for one direction. To further verify this result, we annealed a control W(110) sample with the $I=12$ A down-step current and examined the flip side of the sample where the same current was oriented in the up-step direction. Both the top and the flip side underwent a SB transformation (Fig. 8), although with a higher edge roughness on the flip side due to a coarser surface finish after preparation.

Notably, the grain size in polycrystalline wires, including tungsten, can reach hundreds of $\mu \mathrm{m}$ [97] while the widths of step bunches reach a few $\mu \mathrm{m}$. Potentially, roughening of grain surfaces by SB could be a contributing factor in the formation of voids and failures of hot filament electronic devices (such as electron and ion sources, deposition sources, pressure gauges, and incandescent light bulbs) which are currently attributed solely to electron scattering at the grain boundaries and other lattice defects resulting in the directional displacement of atoms.

The presence of the SB instability on W(110) for both opposing current directions can be explained by changes induced by the electron wind to the transparency (permeability) of atomic steps. This assumption is made on the basis of a theoretical framework developed for $\mathrm{Si}(111)$ where step transparency determines the destabilizing current direction. The current reversals for $\mathrm{Si}(111)$ are assumed to originate from temperature-dependent changes in the step transparency which is determined by density of kink sites along atomic steps [98]. The steps are assumed to be nontransparent when the density of kinks is high in the generalized Burton-CabreraFrank (BCF) theory $[99,100]$, which describes SB induced by down-step atom electromigration $[41,80]$. The steps are described as transparent when the density of kinks is low and, in this case, SB takes place only for up-step atom electromigration [42,101,102]. A recently developed numerical model relies on the assumption that the net electromigration on W(110) coincides with the direction of the electric current (opposite to the electron wind) [95]. At high temperature the atomic steps on W(110) are proposed to be transparent and the 

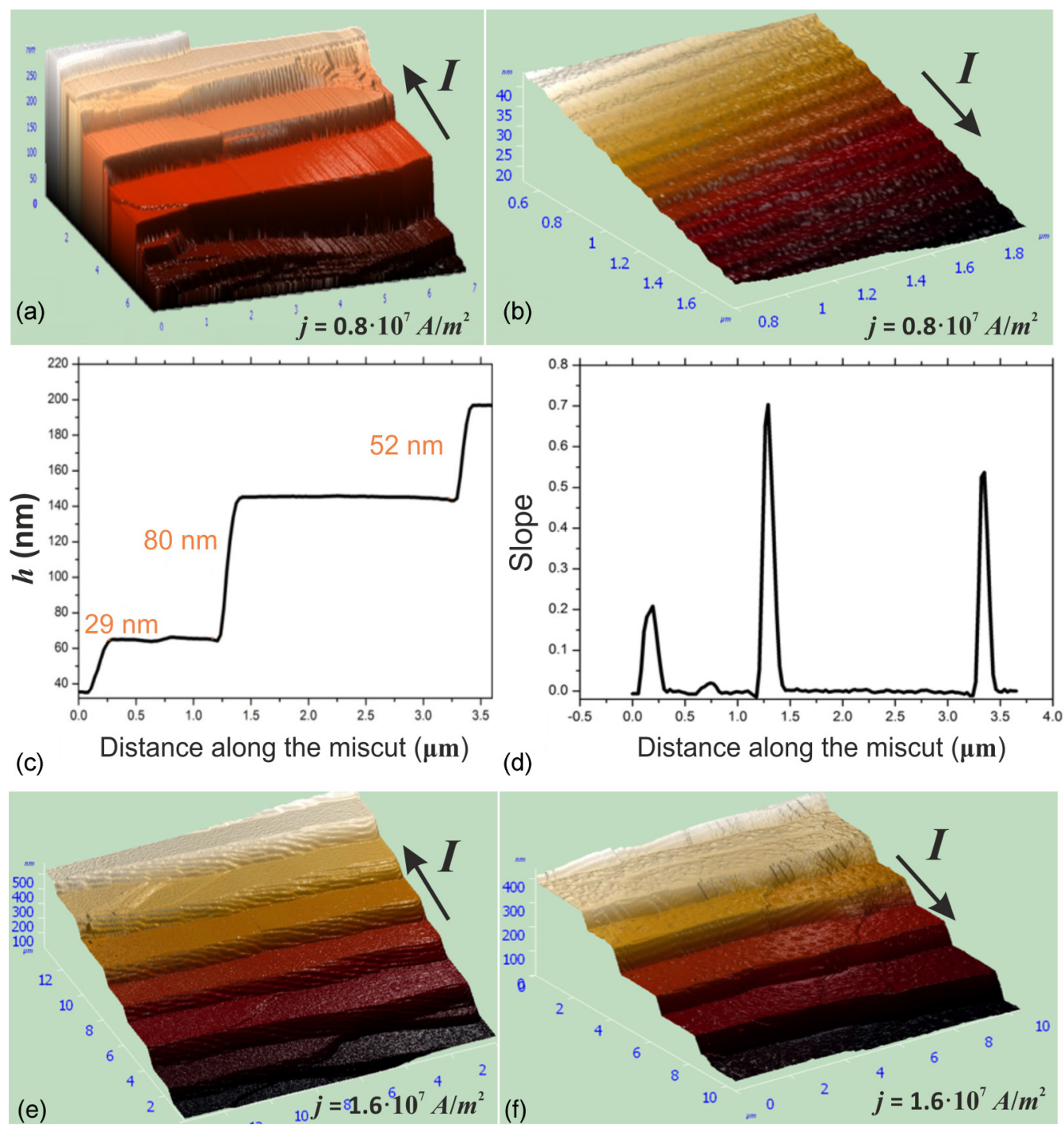

FIG. 7. (a) $7.0 \times 7.0 \times 0.3-\mu \mathrm{m}$ AFM image of a bunched $\mathrm{W}(110)$ surface annealed at $1500{ }^{\circ} \mathrm{C}$ with $I=6 \mathrm{~A} I=6 \mathrm{~A}\left(j=0.8 \times 10^{7} \mathrm{~A} / \mathrm{m}^{2}\right)$ in the up-step direction $\left(P=5 \times 10^{-9}\right.$ Torr $)$. (b) $1.8 . \times 1.8 \times 0.02-\mu \mathrm{m}$ AFM image of W(110) after annealing with $I=6$ A in the down-step direction. Annealing transformed the surface into a monatomic step-density wave. (c) Cross-sectional profile and (d) slope variation along the miscut of the bunched W(110) surface shown in Fig. 7(a). (e) $14.0 \times 14.0 \times 0.5-\mu \mathrm{m}$ AFM image of a bunched W(110) surface created by annealing with $I=12 \mathrm{~A}\left(j=1.6 \times 10^{7} \mathrm{~A} / \mathrm{m}^{2}\right)$ in the up-step direction. (f) $10.0 \times 10.0 \times 0.4-\mu \mathrm{m}$ AFM image of a bunched W(110) surface created by annealing with $I=12 \mathrm{~A}$ in the down-step direction. At present, the surface instability against SB for both up- and down-step currents is unique to $\mathrm{W}(110)$.

surface is unstable against SB for the up-step current, similar to $\mathrm{Si}(111)$. For the down-step current a sufficiently strong electron wind can erode the step edges and increase the kink density to a degree where the steps lose their transparency and take part in the SB. Although calculations based on this model correctly reproduce SB for both up- and down-step directions of electromigration, its basic assumption of net atomic drift in the direction of the applied electric field is at odds with general understandings of electromigration in metals. A possible way to rectify this difference, such that it is consistent with the theory of electromigration in metals, can be found in changes of the atomic step transparency produced by biased atom diffusion along the miscut (which in the case of $\mathrm{Si}(111)$ is realized by changes in temperature [11]). It is known that a down-step diffusional bias results in spontaneous deviations from equilibrium step wandering, while an up-step bias straightens step edges [103]. Considering this for W(110), a down-step current of sufficient strength causing diffusion in the up-step direction could straighten atomic steps (reduce the density of kinks) making them transparent to the adatom flow, thus creating the conditions necessary to destabilize the surface against SB.

Another explanation for the dual direction SB on W(110) we can propose is based on the assumption that SB can proceed universally by either up- or down-step electromigration but different strength of electromigration force is required for the opposing directions. In other words, the value of the critical force, below which the SB ceases, is different for the up- and down-step directions. This assumption is made on the basis of previous studies, which revealed that qualitatively the same morphology is created on $\mathrm{Si}(111)$ by annealing with $E$ fields below critical $\left(E_{\text {cr }}\right)$ and any studied field applied in 

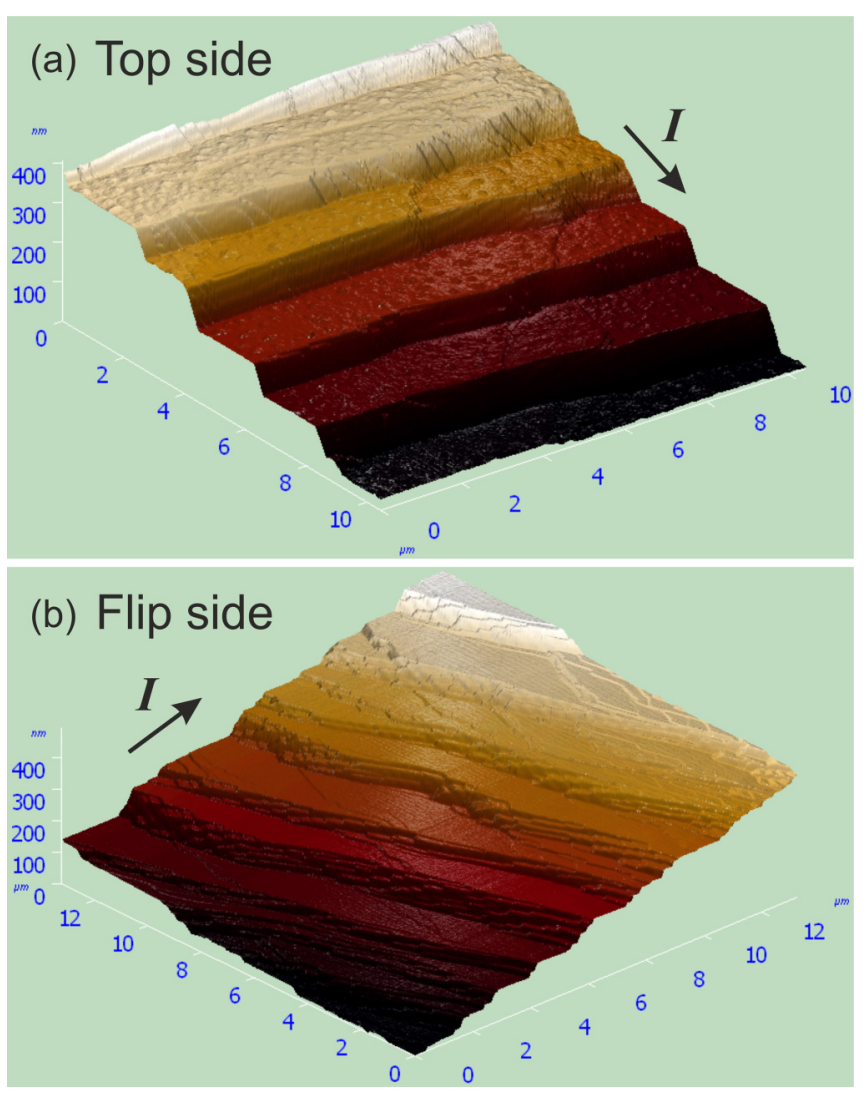

FIG. 8. W(110) instability against SB for both up- and down-step currents demonstrated on the opposite sides of a W(110) rectangular crystal. (a) $10.5 \times 10.5 \times 0.4-\mu \mathrm{m}$ AFM image of the stepbunched top side after annealing at $1500{ }^{\circ} \mathrm{C}$ with $I=12 \mathrm{~A}(j=$ $\left.1.6 \times 10^{7} \mathrm{~A} / \mathrm{m}^{2}\right)$ in the down-step direction relative to the atomic staircase. (b) $13.0 \times 13.0 \times 0.5-\mu \mathrm{m}$ AFM image of the flip side of the same W(110) sample where the same current was oriented in the up-step direction. Both the top and the flip side underwent a SB transformation, although with a loss of the edge straightness on the flip side due to a coarser 600-grit surface finish vs 2000-grit finish on the top.

the opposing direction to where the surface was found to be stable against SB [81]. Both morphologies are characterized by formation of compressed step density waves with relatively small numbers of steps, which are not affected by duration of the annealing. Likewise, on sapphire, similarly faceted surfaces stable against prolonged annealing were created on $\mathrm{Al}_{2} \mathrm{O}_{3}(0001)$ at $1500^{\circ} \mathrm{C}$ by an up-step $E=30 \mathrm{~V} / \mathrm{cm}$ field below critical and the down-step field of $140 \mathrm{~V} / \mathrm{cm}$.

Applying an $E$ field sufficient to reach $E_{\mathrm{cr}}$ for the directions known to be stable against SB is not trivial in the case of $\mathrm{Si}$, as a marginal increase of the electric field is coupled to an exponential growth in joule heating, which will result in a transition to the next temperature regime where $\operatorname{Si}(111)$ is established to be unstable against SB or the melting point is reached. Potentially, this difficulty can be overcome by simultaneous DC annealing and intensive cooling thus reaching a higher electric field at the desired temperature. At the same time, our proposition of different up- and down-step $E_{\mathrm{cr}}$ values for SB could be tested through future work on dielectrics
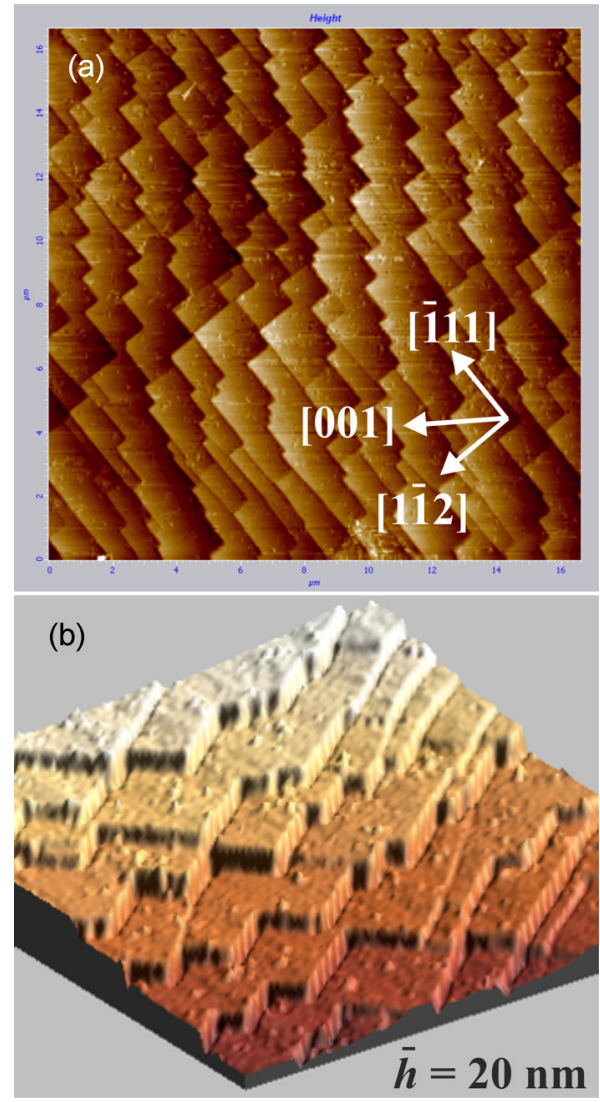

FIG. 9. Effect of the offcut crystallographic alignment on SB. (a) $17.0 \times 17.0-\mu \mathrm{m}$ AFM image of a bunched W(110) surface offcut along the [001] direction and annealed at $1500^{\circ} \mathrm{C}$ with $I=6 \mathrm{~A}$ in the up-step direction. (b) $8.0 \times 8.0 \times 0.12-\mu \mathrm{m} 3 \mathrm{D}$ AFM image of the same surface. Annealing produced morphology of $\sim 20$-nm-high zigzag bunches with the zigzag segments aligned in the [1-12] and [-111] directions making right angle corners pointing in the [001] direction.

where large electric fields can be readily applied, potentially reaching $E_{\mathrm{cr}}$ for both directions.

The crystallographic alignment of the offcut can have considerable impact on SB and may lead to previously unknown surface morphologies. For example, aligning the offcut along the [001] direction on W(110) instead of [1-1-2] and annealing at $1500{ }^{\circ} \mathrm{C}$ while passing a $I=6$ A current in the up-step direction transformed the monatomic topography into a landscape of $\sim 20$-nm-high step bunches with a periodicity of $11.5 \mu \mathrm{m}$ along the offcut (Fig. 9). Noticeably, the bunch edges are composed of zigzag segments aligned in the [1-12] and [-111] directions making right angle corners pointing in the [001] direction which is in stark contrast to the straight bunch edges formed on the [1-1-2] offcut surface (Fig. 7). Clearly, the step edges divert their alignment towards directions of higher atomic density (lower step energy), while avoiding the alignment along the initial lower density [1-10] direction. Notably, the atomic densities along the [-111], [001], and [1-10] directions are $2 / a \sqrt{3}, \frac{1}{a}$, and $1 / a \sqrt{2}$ respectively, where $a=0.316 \mathrm{~nm}$ is the tungsten lattice constant. This morphology is likely created by a combination of SB and thermal anisotropic diffusion related to the anisotropy of the 
surface crystallographic structure. On the one hand, the SB proceeds by atomic exchange between the steps by means of the down-step $E$-field biased migration, while on the other hand, preferential diagonal atom diffusion along more closely spaced hopping sites in the [1-12] and [-111] directions aligns step edges along these paths. As a result, the steps agglomerate into step bunches while diverting their alignment away from the [1-10] direction, bestowing the step bunches their zigzag shape.

Visibly similar morphologies at a smaller scale can be obtained on W(110) substrates, offcut along the [001] direction, for submonolayer films of transition metals. Specifically, submonolayer-thick Fe films order upon annealing at $700{ }^{\circ} \mathrm{C}$ into monolayer-thick triangles alongside the atomic steps [19]. The triangles form continuous zigzag edges with sides symmetric about the [001] offcut axis, aligned in the [1-12] and $[-112]$ directions and forming a $70.5^{\circ}$ angle, indicating the energetically favorable paths for $\mathrm{Fe}$ atoms attachment on $\mathrm{W}(110)$. This contrasts with the bunches on $\mathrm{W}(110)$ where the sides are aligned in the [1-12] and [-111] directions, making the right-angle corners asymmetric about the [001] axis. It is established that thermal annealing alone is insufficient to realign the atomic steps on monoelemental $\mathrm{W}(110)$ or induce three-dimensional SB growth. However, it is unknown at what stage during electromigration this realignment of atomic steps occurs on $\mathrm{W}(110)$, akin to the heteroepitaxial Fe/W(110) system. To probe this aspect of step evolution, time resolved annealing, or reduced current density measurements would be desirable.

\section{CONCLUSIONS}

Electromigration induced step bunching has been revealed on dielectric and metallic vicinal surfaces and may be more prevalent than previously assumed. Electric fields applied at $1500{ }^{\circ} \mathrm{C}$ transformed surface morphologies of vicinal $\mathrm{Al}_{2} \mathrm{O}_{3}(0001)$ and $\mathrm{W}(110)$, so they closely resembled that of $\mathrm{Si}(111)$ step bunched by electromigration. Common features include, in particular, the scaling relationships between the step bunch height and its slope, atomic crossing steps, antibands, the existence of a SB cutoff critical field, and dependence of the end surface morphology on the miscut direction.
SB instability on $\mathrm{Al}_{2} \mathrm{O}_{3}(0001)$ was induced at $1500^{\circ} \mathrm{C}$ by electric fields ranging between 70 and $140 \mathrm{~V} / \mathrm{cm}$ applied along the [1-210] miscut in the up-step direction. Annealing with an $E$ field of $30 \mathrm{~V} / \mathrm{cm}$ created a faceted surface which was reproduced by annealing with the $E$ fields oriented in the down-step direction or by thermal annealing alone. The critical field in the range $30-70 \mathrm{~V} / \mathrm{cm}$ is at least a factor of 60 greater than $E_{\text {cr }}$ for the up-step electromigration on $\mathrm{Si}(111)$ indicating either lower mobility or lower effective charge (or both) of surface adatoms on $\mathrm{Al}_{2} \mathrm{O}_{3}(0001)$. We suggest that $\mathrm{SB}$ on $\mathrm{Al}_{2} \mathrm{O}_{3}(0001)$ is driven by direct electrostatic force acting on uncompensated positive charges of $\mathrm{Al}$ surface atoms, as seen on $\mathrm{Si}(111)$.

Significant direct forces could not be applied in the case of tungsten, as the samples acted as effective shortcuts due to tungsten low resistivity ( $E=0.04$ or $0.08 \mathrm{~V} / \mathrm{cm}$ along the miscut in the [1-1-2] direction). On this ground we suggest that the electron wind was a driving force responsible for SB on $\mathrm{W}(110)$ due to a large effective charge of tens of elementary charges which could be locally enhanced for atoms at the step-edge and kink positions. Annealing W(110) offcut along the [001] direction with the up-step current produced step bunches with the edges composed of zigzag segments aligned in the [1-12] and [-111] directions making right angle corners pointing in the [001] direction. We suggest that this previously unobserved morphology was likely created by a combination of SB and thermal anisotropic diffusion related to the anisotropy of the surface crystallographic structure.

The expansion of the scope of electromigration assisted SB to include dielectrics and metals opens opportunities for future experimental and theoretical studies. This provides media to comprehensively assess surface processes and transformations. It opens an opportunity for future work on surface tailoring, functionalization, and transformation, potentially creating different surface morphologies and reconstructions, unattainable merely by means of thermal, mechanical, or chemical surface treatment.

\section{ACKNOWLEDGMENTS}

We acknowledge financial support of Irish Research Council Laureate Award No. IRCLA/2019/171. O.T. acknowledges support of the government of Kazakhstan under the Bolashak program.
[1] R. A. Reisfeld, U. J. Lewis, and D. E. Williams, Nature (London) 195, 281 (1962).

[2] M. Ramos-Payan, J. A. Ocana-Gonzalez, R. M. FernandezTorres, A. Llobera, and M. A. Bello-Lopez, Electrophoresis 39, 111 (2018).

[3] R. Waser and M. Aono, Nat. Mater 6, 833 (2007).

[4] V. K. Sangwan, H. S. Lee, H. Bergeron, I. Balla, M. E. Beck, K. S. Chen, and M. C. Hersam, Nature (London) 554, 500 (2018).

[5] I. Zhitomirsky, Adv. Colloid Interface Sci. 97, 279 (2002).

[6] P. Krishnakumar, P. B. Tiwari, S. Staples, T. Luo, Y. Darici, J. He, and S. M. Lindsay, Nanotechnology 23, 455101 (2012).
[7] N. Wang, K. H. Bevan, and N. Provatas, Phys. Rev. Lett. 117, 155901 (2016).

[8] J. R. Black, IEEE Trans. Electron Devices. 16, 338 (1969).

[9] J. Lienig and M. Thiele, Fundamentals of Electromigration-Aware Integrated Circuit Design (Springer, 2018).

[10] M. Degawa, H. Minoda, Y. Tanishiro, and K. Yagi, Surf. Sci. 461, L528 (2000).

[11] A. Latyshev, A. Aseev, A. Krasilnikov, and S. Stenin, Surf. Sci. 213, 157 (1989).

[12] J. J. De Yoreo, Principles of Crystal Growth and Nucleation (Mineralogical Society of America, Napa, CA, 2003). 
[13] X. Gong, Nat. Mater. 5665 (2006).

[14] C. Hahn, J. Shan, Y. Liu, O. Berg, A. W. Kleijn, and L. B. Juurlink, J. Chem. Phys. 136, 114201 (2012).

[15] C. Tegenkamp, J. Phys.: Condens. Matter 21, 013002 (2009).

[16] G. A. Somorjai and D. W. Blakely, Nature (London) 258, 580 (1975).

[17] F. Cuccureddu, V. Usov, S. Murphy, C. O'Coileain, and I. V. Shvets, Rev. Sci. Instrum. 79, 053907 (2008).

[18] S. Murphy, D. Mac Mathuna, G. Mariotto, and I. V. Shvets, Phys. Rev. B 66, 195417 (2002).

[19] H. J. Elmers, J. Hauschild, and U. Gradmann, J. Magn. Magn. Mater. 221, 219 (2000).

[20] V. Usov, S. Murphy, and I. V. Shvets, J. Magn. Magn. Mater. 286, 18 (2005).

[21] S. Dahl, A. Logadottir, R. C. Egeberg, J. H. Larsen, I. Chorkendorff, E. Törnqvist, and J. K. Nørskov, Phys. Rev. Lett. 83, 1814 (1999).

[22] R. T. Vang, K. Honkala, S. Dahl, E. K. Vestergaard, J. Schnadt, E. Lægsgaard, B. S. Clausen, J. K. Nørskov, and F. Besenbacher, Nat. Mater. 4, 160 (2005).

[23] B. L. M. Hendriksen, M. D. Ackermann, R. van Rijn, D. Stoltz, I. Popa, O. Balmes, A. Resta, D. Wermeille, R. Felici, S. Ferrer, and J. W. M. Frenken, Nat. Chem. 2, 730 (2010).

[24] V. Usov, S. Murphy, and I. V. Shvets, J. Magn. Magn. Mater. 283, 357 (2004).

[25] H. C. Mireles and J. L. Erskine, Phys. Rev. Lett. 87, 037201 (2001).

[26] J. Chen and J. L. Erskine, Phys. Rev. Lett. 68, 1212 (1992).

[27] H. J. Choi, Z. Q. Qiu, J. Pearson, J. S. Jiang, D. Li, and S. D. Bader, Phys. Rev. B 57, R12713(R) (1998).

[28] W. Weber, C. H. Back, A. Bischof, Ch. Würsch, and R. Allenspach, Phys. Rev. Lett. 76, 1940 (1996).

[29] J.V. Barth, G. Costantini, and K. Kern, Nature (London) 437, 671 (2005).

[30] T. Zubkov, G. A. Morgan, Jr., J. T. Yates, Jr., O. Kuhlert, M. Lisowski, R. Schillinger, D. Fick, and H. J. Jänsch, Surf. Sci. 526, 57 (2003).

[31] M. Sprinkle, M. Ruan, Y. Hu, J. Hankinson, M. Rubio-Roy, B. Zhang, X. Wu, C. Berger, and W. A. de Heer, Nat. Nanotechnol. 5, 727 (2010).

[32] A. Sgarlata, P. D. Szkutnik, A. Balzarotti, N. Motta, and F. Rosei, Appl. Phys. Lett. 83, 4002 (2003).

[33] O. Ualibek, R. Verre, B. Bulfin, V. Usov, K. Fleischer, J. F. McGilp, and I. V. Shvets, Nanoscale 5, 4923 (2013).

[34] I. V. Shvets, H. C. Wu, Usov F. Cuccureddu, S. K. Arora, and S. Murphy, Appl. Phys. Lett. 92, 023107 (2008).

[35] J. J. Métois, A. Saúl, and P. Müller, Nat. Mater. 4, 238 (2005).

[36] S. Park, D. H. Lee, J. Xu1, B. Kim, S. W. Hong, U. Jeong, T. Xu, and T. P. Russell, Science 323, 1030 (2009).

[37] A. A. Shklyaev and A. V. Latyshev, Appl. Surf. Sci. 465, 10 (2019).

[38] V. Usov, C. Ó Coileáin, and I. V. Shvets, Phys. Rev. B 83, 155321 (2011).

[39] K. Fujita, M. Ichikawa, and S. S. Stoyanov, Phys. Rev. B 60, 16006 (1999).

[40] Y. Homma and N. Aizawa, Phys. Rev. B 62, 8323 (2000).

[41] S. Stoyanov and V. Tonchev, Phys. Rev. B 58, 1590 (1998).

[42] S. Stoyanov, Surf. Sci. 416, 200 (1998).

[43] F. Krzyiewski, M. Zaluska-Kotur, A. Krasteva, H. Popova, and V. Tonchev, Cryst. Growth Des. 19, 821 (2019).
[44] V. Usov, C. Ó Coileáin, and I. V. Shvets, Phys. Rev. B 82, 153301 (2010).

[45] M. Gautier-Soyer, F. Jollet, and C. Noguera, Surf. Sci. 352, 755 (1996).

[46] C. Sousa, F. Illas, and G. Pacchioni, J. Chem. Phys. 99, 6818 (1993).

[47] C. Ruberto, Y. Yourdshahyan, and B. I. Lundqvist, Phys. Rev. B 67, 195412 (2003).

[48] R. Di Felice and J. E. Northrup, Phys. Rev. B 60, R16287 (1999).

[49] X. G. Wang, A. Chaka, and M. Scheffler, Phys. Rev. Lett. 84, 3650 (2000).

[50] I. G. Batyrev, A. Alavi, and M. W. Finnis, Phys. Rev. B 62, 4698 (2000).

[51] Z. Lodziana, J. K. Norskov, and P. Stoltze, J. Chem. Phys. 118, 11179 (2003)

[52] A. Marmier and S. C. Parker, Phys. Rev. B 69, 115409 (2004).

[53] P. Gue'nard, G. Renaud, A. Barbier, and M. Gautier-Soyer, Surf. Rev. Lett. 5, 321 (1997).

[54] J. Ahn and J. W. Rabalais, Surf. Sci. 388, 121 (1997).

[55] T. Suzuki, S. Hishita, K. Oyoshi, and R. Souda, Surf. Sci. 437, 289 (1999).

[56] J. Guo, D. E. Ellis, and D. J. Lam, Phys. Rev. B 45, 13647 (1992).

[57] C. Rohmann, J. B. Metson, and H. Idriss, Surf. Sci. 605, 1694 (2011).

[58] H. J. Monkhorst and J. D. Pack, Phys. Rev. B 13, 5188 (1976).

[59] C. Verdozzi, D. R. Jennison, P. A. Schultz, and M. P. Sears, Phys. Rev. Lett. 82, 799 (1999).

[60] E. A. A. Jarvis and E. A. Carter, J. Phys. Chem. B 105, 4045 (2001).

[61] P. W. Tasker, in Structure and Properties of $\mathrm{MgO}$ and $\mathrm{Al}_{2} \mathrm{O}_{3}$ Ceramics, edited by W. D. Kingery, Advances in Ceramics (American Ceramic Society, Columbus, 1984), Vol. 10, p. 176.

[62] J. Goniakowski, F. Finocchi, and C. Noguera, Rep. Prog. Phys. 71016501 (2008).

[63] C. C. Chang, J. Appl. Phys. 39, 5570 (1968).

[64] T. M. French and G. A. Somorjai, J. Phys. Chem. 74, 2489 (1970).

[65] J. Toofan and P. R. Watson, Surf. Sci. 401, 162 (1998).

[66] I. Vilfan, T. Deutsch, F. Lancon, and G. Renaud, Surf. Sci. 505, L215 (2002).

[67] J. V. Lauritsen and M. Reichling, J. Phys.: Condens. Matter 22, 263001 (2010).

[68] J. Wang, A. Howard, R. G. Egdell, J. B. Pethica, and J. S. Foord, Surf. Sci. 515, 337 (2002).

[69] G. Renaud, B. Villette, I. Vilfan, and A. Bourret, Phys. Rev. Lett. 73, 1825 (1994).

[70] J. M. Charig, Appl. Phys. Lett. 10, 139 (1967).

[71] G. Renaud, Surf. Sci. Rep. 32, 5 (1998).

[72] A. Tosaka, T. Kitamura, T. Sugiyama, K. Koyama, and Y. Shigeta, Appl. Phys. Lett. 104, 221601 (2014).

[73] C. Barth and M. Reichling, Nature (London) 414, 54 (2001).

[74] J. V. Lauritsen, M. C. R. Jensen, K. Venkataramani, B. Hinnemann, S. Helveg, B. S. Clausen, and F. Besenbacher, Phys. Rev. Lett. 103, 076103 (2009).

[75] I. Vilfan, F. Lancon, and J. Villain, Surf. Sci. 392, 62 (1997).

[76] C. L. Pang, H. Raza, S. A. Haycock, and G. Thornton, Surf. Sci. 460, L510 (2000). 
[77] M. Gautier, G. Renaud, L. Pham Van, B. Villette, M. Pollak, N. Thromat, F. Jollet, and J.-P. Duraud, J. Am. Ceram. Soc. 77, 323 (1994).

[78] J. R. Heffelfinger, M. W. Bench, and C. B. Carter, Surf. Sci. 370, L168 (1997).

[79] R. Verre, R. G. S. Sofin, V. Usov, K. Fleischer, D. Fox, G. Behan, H. Zhang, and I. V. Shvets, Surf Sci. 606, 1815 (2012).

[80] J. Krug, V. Tonchev, S. Stoyanov, and A. Pimpinelli, Phys. Rev. B 71, 045412 (2005).

[81] C. Ó Coileáin, V. Usov, I. V. Shvets, and S. Stoyanov, Phys. Rev. B 84, 075318 (2011).

[82] A. Latyshev, A. Krasilnikov, and A. Aseev, Surf. Sci. 311, 395 (1994).

[83] E. E. Rodyakina, S. S. Kosolobov, and A. V. Latyshev, JETP Lett. 94, 147 (2011).

[84] V. Usov, S. Stoyanov, C. O Coileain, O. Toktarbaiuly, and I. V. Shvets, Phys. Rev. B 86, 195317 (2012).

[85] K. Thurmer, D. J. Liu, E. D. Williams, and J. D. Weeks, Phys. Rev. Lett. 83, 5531 (1999).

[86] K. Yagi, H. Minoda, and M. Degawa, Surf. Sci. Rep. 43, 45 (2001).

[87] V. A. Sorkin, Tungsten Technical Data (Technical Report of VNIITS, Moscow, 1988).

[88] R. S. Sorbello, Phys. Rev. B 31, 798 (1985).

[89] B. Chao, S. H. Chae, X. Zhang, K. H. Lu, J. Im, and P. S. Ho, Acta Mater. 55, 2805 (2007).

[90] M. F. G. Hedouin and P. J. Rous, Phys. Rev. B 62, 8473 (2000).
[91] T. J. Stasevich, C. G. Tao, W. G. Cullen, E. D. Williams, and T. L. Einstein, Phys. Rev. Lett. 102, 085501 (2009).

[92] Z. Yang and M. Di Ventra, Phys. Rev. B 67, 161311(R) (2003).

[93] C. Tao, W. G. Cullen, and E. D. Williams, Science 328, 736 (2010).

[94] M. Rusanen, P. Kuhn, and J. Krug, Phys. Rev. B 74, 245423 (2006).

[95] O. Toktarbaiuly, V. Usov, C. Ó Coileáin, K. Siewierska, S. Krasnikov, E. Norton, S. I. Bozhko, V. N. Semenov, A. N. Chaika, B. E. Murphy, O. Lübben, F. Krzyzewski, M. A. Załuska-Kotur, A. Krasteva, H. Popova, V. Tonchev, and I. V. Shvets, Phys. Rev. B 97, 035436 (2018).

[96] K. Radican, S. I. Bozhko, S. R. Vadapoo, S. Ulucan, H. C. Wu, A. Mccoy, and I. V. Shvets, Surf. Sci. 604, 1548 (2010).

[97] Q. Wei and L. J. Kecskes, Mater. Sci. Eng. A 491, 62 (2008).

[98] S. Filimonov and Y. Hervieu, Surf. Sci. 553, 133 (2004).

[99] W. Burton, N. Cabrera, and F. C. Frank, Philos. Trans. R. Soc. London, Ser. A 243, 299 (1951).

[100] S. Stoyanov, Jpn. J. Appl. Phys. Part 130, 1 (1991).

[101] P. Nozieres, in Solids Far from Equilibrium, edited by C. Godr'eche (Cambridge University Press, Cambridge, UK, 1991).

[102] S. Stoyanov, Surf. Sci. 370, 345 (1997).

[103] R. Yongsunthon, C. G. Tao, P. Rous, and E. D. Williams, Nanophenomena at Surfaces, Springer Series in Surface Science Vol. 47 (Springer, Berlin, 2011), p. 113. 